\title{
Congenital Reticular Ichthyosiform Erythroderma
}

V. Dvorakova, ${ }^{1}$ RM Watson, ${ }^{1}$ A. Terron-Kwiatkowski, ${ }^{2}$ N. Andrew ${ }^{2}$ and AD. Irvine ${ }^{1,3,4}$

${ }^{1}$ Department of Dermatology, Our Lady's Children's Hospital, Crumlin, Dublin, Ireland; 'East of Scotland Regional Molecular Genetics Service, Human Genetics, Ninewells Hospital and Medical School, Dundee, UK

${ }^{3}$ Clinical Medicine, Trinity College Dublin, ${ }^{4}$ National Children's Research Centre, Crumlin, Ireland

Corresponding author: Alan Irvine, Department of Dermatology, Our Lady's Children's Hospital, Crumlin, Dublin, Ireland. IRVINEA@tcd.ie

Keywords: ichthyosis with confetti, keratin 10, spontaneous mitotic recombination, Congenital Reticular Ichthyosiform Erythroderma

Word count: 674

Figure count: 2

Conflict of interest: the authors declare that they have no conflict of interest. 
Ichthyosis with confetti (IWC) (OMIM 609165), also known as congenital reticular ichthyosiform erythroderma, is a rare autosomal dominant ichthyosis caused by heterozygous mutations in the keratin 10 (KRT10) gene. ${ }^{1}$ Affected individuals present with erythroderma and scaling at birth but gradually develop multiple characteristic confetti-like clear areas of normal skin throughout their childhood. Diagnosis of IWC is therefore often delayed until early adolescence when areas of normal appearing skin become larger and more numerous.

A female infant was born via spontaneous vaginal delivery at 36 weeks gestation to non-consanguineous parents. Erythroderma with scaling was noted at birth in the absence of bullae or collodion membrane. The baby had low set small ears, small nares and normal nails. Skin biopsy from the abdomen showed mild parakeratosis and acanthosis, increased basal and parabasal mitotic activity and non-specific spongiosity. Hair analysis was normal on light and electron microscopy. The overall clinical picture was felt to be consistent with congenital ichthyosiform erythroderma (CIE). Her family then moved abroad and the patient was followed in a different dermatology institution. Her early course was complicated by recurrent Staphylococcus aureus infections. The ichthyosis was managed with $0.5 \mathrm{mg} / \mathrm{kg}$ of oral etretinate between the ages 2 - 4 and a trial of topical tretinoin aged 5 . Bilateral ear canal impaction with squamous debris required repeated suctioning and the patient also underwent successful release of bilateral cryptotia age 4. Excessive hair growth on the dorsal part of limbs as well as pubic area was investigated at age 3 with no underlying hormonal abnormality identified at the time. 
The patient subsequently returned for follow up with our service at the age of 9 . Clinical examination revealed short stature, ichthyosis, erythroderma and multiple confetti areas of normal skin predominantly on trunk and face (Fig.1) Palmoplantar keratoderma was now more obvious and the patient also developed bilateral ectropion and flexion contractures of her neck and elbows( Fig.2). Recurrent skin infections, both bacterial and fungal in origin, continued to complicate her course. The patient is now 21 years old and recently underwent successful correction of a right eye ectropion with skin graft from inner thigh area. Recent DEXA scan showed osteopaenia and repeat thyroid function tests revealed subclinical hypothyroidism with mildly elevated TSH and patient was commenced on low dose levothyroxine and dietary calcium supplements. Her ichthyosis and palmoplantar keratoderma are managed only with emollients at present.

In light of recent reports ${ }^{1,2}$ we then screened the patients genomic DNA extracted from PBMCs for mutations in the coding sequence of $K R T 10$ gene and identified a heterozygous c.1374-1G>C mutation at the acceptor splice site of exon 7 . This splice site mutation has previously been reported ${ }^{3}$ and is predicted to result in aberrant keratin 10 , most likely by the use of an alternative cryptic acceptor splice site within exon 7 resulting in a frameshift and an arginine-rich tail domain. Previously reported mutations causing IWC were shown to create frameshifts in intron 6 or exon 7 of KRT10 resulting in arginine rich tails. ${ }^{1,2}$

The first report of the genetic basis for IWC ${ }^{1}$ identified heterozygous frameshift mutations in the KRT10 gene resulting in arginine-rich C-terminal peptide as the cause of IWC. This premature stop is predicted to result in mislocalisation of keratin 10 to the nucleolus, disrupting the keratin network and leading to loss of barrier function. This mutation was lost due to correction through revertant mosaicism in 
confetti-like areas of normal skin. Another disorder associated with mutations in keratin 10 gene is epidermolytic ichthyosis (EI), previously known as epidermolytic hyperkeratosis. KRT10 mutations reported in El however do not result in arginine rich tail as is the case in IWC. Our case further confirms that specific KRT10 gene defects affecting the keratin 10 tail cause IWC and also highlights the typical initial presentation with erythroderma and scaling resembling CIE with gradual development of areas of healthy skin within ichthyotic skin during childhood and early adolescence. Further clinical features present in our patient and similar to other reports of IWC include prominent palmoplantar keratoderma, hypertrichosis affecting dorsal limbs ${ }^{2,3,4,5}$, ectropion ${ }^{2,3,4,5}$, short stature ${ }^{3,5}$, but also contraction neck deformity and cryptotia ${ }^{3}$.

\section{References}

1. Choate KA, Lu Y, Zhou J et al. Mitotic recombination in patients with ichthyosis causes reversion of dominant mutations in KRT10. Science. 2010; $330: 94-97$.

2. Burger B, Spoerri I, Schubert M et al. Description of the natural course and clinical manifestations of ichthyosis with confetti caused by a novel KRT10 mutation. Br J Dermatol 2012;166(2):434-439.

3. Spoerri, I, Brena, M, De Mesmaeker, J et al. The phenotypic and genotypic spectra of ichthyosis with confetti plus novel genetic variation in the 3 ' end of KRT10: from disease to a syndrome. JAMA Dermatol.2015; 151(1):64-69.

4. Brusasco A, Tadini G, Cambiaghi S et al. A case of congenital reticular ichthyosiform erythroderma: ichthyosis "en confettis".Dermatology 1994; 188 : $40-45$. 
5. Diociaiuti, A., P. Fortugno, M. El Hachem et al. "Early immunopathological diagnosis of ichthyosis with confetti in two sporadic cases with new mutations in keratin 10." Acta Derm Venereol 2014;94(5):579-82. 
Figure 1 (a) Chest and (b) back confetti-like areas of normal skin.

Figure 2 (a) dorsal and (b) ventral side of arm with hyperkeratotic palm, elbow contracture and cofetti - like areas of normal skin. 


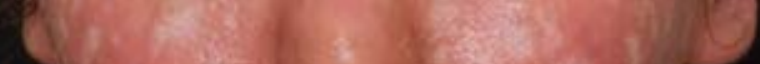




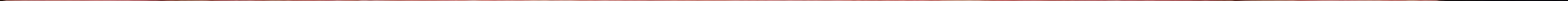



